\title{
Dairy Consumption and Risk of Conventional and Serrated Precursors of Colorectal Cancer: A Systematic Review and Meta-Analysis of Observational Studies
}

\author{
Li-Liangzi Guo $\mathbb{D},{ }^{1,2}$ Yu-Ting Li $\mathbb{D},{ }^{1}$ Jun Yao $\mathbb{D}^{2},{ }^{2}$ Li-Sheng Wang $\mathbb{D}^{2},{ }^{2}$ Wei-Wei Chen $\mathbb{D}^{1}{ }^{1}$ \\ Kai-Yin He $\mathbb{D}^{1}{ }^{1}$ Lin Xiao $\mathbb{D}^{1},{ }^{1}$ and Shao-Hui Tang $\mathbb{D}^{1}$ \\ ${ }^{1}$ Department of Gastroenterology, The First Affiliated Hospital, Jinan University, Guangzhou, Guangdong 510632, China \\ ${ }^{2}$ Department of Gastroenterology, Shenzhen People's Hospital (The Second Clinical Medical College, Jinan University, \\ The First Affiliated Hospital, Southern University of Science and Technology), Shenzhen, Guangdong 518020, China
}

Correspondence should be addressed to Shao-Hui Tang; tangshaohui206@163.com

Received 15 March 2021; Revised 29 April 2021; Accepted 7 May 2021; Published 26 May 2021

Academic Editor: Akira Hara

Copyright $\odot 2021$ Li-Liangzi Guo et al. This is an open access article distributed under the Creative Commons Attribution License, which permits unrestricted use, distribution, and reproduction in any medium, provided the original work is properly cited.

Objective. The consumption of dairy is associated with decreased risk of colorectal cancer (CRC), but few studies have assessed the relationship between dairy consumption and precursors of CRC. Therefore, we performed the first meta-analysis to further evaluate this association. Methods. PubMed, Embase, Scopus, and Web of Science databases were searched through July 2020 for observational studies. Study-specific risk estimates for the highest versus lowest category were pooled using the random-effects and fixed-effects model. The methodological quality of included studies was assessed using the ROBINS-I Scale. Results. A total of 12 studies were included ( 3 cohort studies and 9 case-control studies). Compared with the lowest level consumption, fermented dairy products had a decreased risk of precursors of CRC in both cohort ( $\mathrm{RR}=0.9295 \% \mathrm{CI}$ : 0.87-0.97) and case-control studies $(\mathrm{RR}=0.98$ 95\% CI: 0.96-0.99). Total dairy $(\mathrm{RR}=0.80$ 95\% CI: 0.68-0.96) and cheese $(\mathrm{RR}=0.96$ 95\% CI: $0.93-0.99)$ consumption was inversely associated with the risk in case-control studies whereas yogurt consumption was inversely associated with the risk in cohort studies ( $R R=0.9195 \% \mathrm{CI}$ : 0.86-0.96). No significant associations were found for consumption of total milk and non/lowfat milk. For dose-response analyses, evidence of linear association was found in total dairy and yogurt consumption. The risk decreased by $12 \%$ for an increment of $200 \mathrm{~g} / \mathrm{d}$ total dairy consumption $(\mathrm{RR}=0.8895 \% \mathrm{CI}$ : $0.81-0.95)$ and decreased by $8 \%$ for an increment of $50 \mathrm{~g} / \mathrm{d}$ yogurt consumption ( $\mathrm{RR}=0.92 \mathrm{95 \%} \mathrm{CI}$ : $0.85-0.99)$. Conclusions. Fermented dairy products, specifically yogurt and cheese, were significantly associated with decreased risk of conventional and serrated precursors of colorectal cancer.

\section{Introduction}

According to GLOBOCAN 2018, colorectal cancer (CRC) is the third most frequently diagnosed cancer and the second leading cause of cancer death. It is estimated that there will be over 1.8 million new CRC cases and 881,000 deaths in 2018 [1]. It is well known that cancer is a complicated disease caused by the interaction of environmental and genetic factors, such as smoking, alcohol, and diet [2]. Various dietary factors have been found to be important protective factors for CRC. For example, the Mediterranean diet might decrease the risk of CRC $[3,4]$. In an umbrella review of observational studies, intake of total dairy was associated with decreased risk of cardiovascular disease, hypertension, and fatal stroke [5]. The latest report of World Cancer Research Fund (WCRF) and American Institute of Cancer Research (AICR) also reached strong evidence that dairy products intake can help protect against CRC [6].

Adenomas (adenomatous polyps, AP) and a subset of serrated lesions (traditional serrated adenoma (TSA) and sessile serrated adenoma/polyp (SSA/P) are two major subtypes of the precursors of CRC. A previous meta-analysis has shown an increased risk of colorectal adenoma (CRA) with the intake of red and processed meat $[7,8]$; a decreased 
risk with increased intake of dietary fiber [9], calcium [10], magnesium [11], selenium, [12] and coffee [13]; and a null association with white meat [14]. However, the relationship between dairy consumption and precursors of CRC is still uncertain. To better understand this relationship, we combined all published epidemiologic studies on the association between dairy intake and precursors of CRC risk and then conducted a dose-response meta-analysis.

\section{Methods}

2.1. Design. The protocol of this meta-analysis was registered in PROSPERO (CRD42020192846). This study was reported according to the Preferred Reporting Items for Systematic Reviews and Meta-Analysis (PRISMA) guidelines [15]. The completed PRISMA checklist is provided online. PICO format (population, intervention, comparison, outcome) was employed to answer the research question: "Are dairy associated with the occurrence of conventional and serrated precursors of colorectal cancer." Population: adults with conventional adenomas (including tubular adenoma, tubulovillous adenoma, villous adenoma, and adenoma with high-grade dysplasia) and serrated precursors of colorectal cancer (TSA and SSA/P); cases with hyperplastic polyps (HP) were excluded from the analyses. Intervention: total dairy product intake (total dairy, milk, cheese, butter, yogurt, or other dairy products). Comparison: adults without colorectal adenoma or serrated lesion. Outcome: the occurrence of conventional adenomas and serrated lesions.

2.2. Search Strategy. We searched PubMed, Embase, Scopus, and Web of Science database in English until April 2020. The following search terms were used: "dairy" or "dairy products" or "fermented milk products" or "cultured milk products" or "fermented dairy products" or subtypes of dairy products (i.e., "milk" or "yogurt" or "yoghurt" or "cheese" or "hard cheese" or "cottage cheese" or "cheddar" or "butter" or "buttermilk" or "cream" or "ice cream" or "Kefir"; NOT: "milk protein" or "whey protein"; AND: "colorectal adenoma" or "colorectal polyp" or "colorectal lesion" or "colorectal neoplasm" or "colorectal tumor" or "colorectal carcinoma" or "colorectal cancer" without restrictions. Titles and abstracts were screened independently by two reviewers (Liliangzi Guo and Yuting Li) to exclude irrelevant articles. Then, the full texts were retrieved to further increase the potentially relevant articles.

2.3. Study Selection. Inclusion criteria: (1) adult participants>18 years of age; (2) cohort studies, case-control studies, or cross-sectional studies that investigated the association between dairy consumption and risk of conventional adenomas and serrated lesions; (3) diagnosis: colorectal adenomas and serrated lesions that were determined by histology; (4) studies that reported the risk estimates (relative risk (RR), odds ratio (OR), or hazard ratio (HR)) with their corresponding 95\% confidence interval (CI) or available original data allowing us to compute the 95\% CI; (5) if the published studies reported data for specific subgroups, results for the whole population were considered in this meta-analysis; (6) if the original publications provided several independent studies, they were considered as separate studies in the following data analysis.

Exclusion criteria: (1) animal studies; (2) studies conducted on children, adolescents, or pregnancy women; (3) nonoriginal papers (reviews, editorials, or commentaries); (4) meta-analysis studies; (5) studies that did not provide enough data on dairy products consumption and risk estimates; (6) duplicate reports and abstracts; and (7) studies that investigated the risk of CRC or the recurrence of colorectal adenomas or serrated lesions.

2.4. Data Extraction and Quality Assessment. The data extracted from each study included the name of first author, publication year, study region, study design, sample size, type of colorectal lesion, dairy products categories, the risk estimates with their 95\% CIs for each category of exposure variables and adjusted covariates in the multivariable analysis. We extracted the risk estimates that reflected the greatest degree of adjustment for potential confounders.

Dose-response analysis requires the distribution of cases and controls, person-years or noncases, and risk estimates with 95\% CIs for at least three quantitative categories of exposure to dairy. The median or mean level of dairy intake for each category was assigned to the corresponding RR for every study. The interval size of the open-ended highest category was assumed as the closest interval while the lowest was considered as zero [9]. Two investigators (Liliangzi Guo and Weiwei Chen) independently extracted the data. Any disagreements were resolved by consulting the third investigator (Shaohui Tang).

Two investigators (Weiwei Chen and Kaiyin $\mathrm{He}$ ) independently assessed the study quality based on the Risk of Bias in Nonrandomized Studies of Interventions (ROBINSI) tool which contains seven domains. According to ROBINS-I guidance, the study judged to be at low risk in all seven domains was considered as low risk of bias; the study judged to be at low or moderate risk in all domains was considered as moderate risk of bias; the study judged to be at serious risk or critical risk in at least one domain was considered as serious risk of bias or critical risk of bias, respectively [16].

The definition of the dairy category (Table S1) in this meta-analysis is listed as follows. Total dairy includes total milk and fermented dairy product. Total milk includes non/ low-fat milk and whole milk. The fermented dairy product includes yogurt, cheese, and cottage cheese.

2.5. Statistical Analysis. The results were expressed in terms of RR and 95\% CI for the highest versus lowest category of dairy products consumption. The standard error of the logarithmic OR/RR of each study was calculated and taken as the estimated variance of the logarithmic OR/RR. The inverse variance method was adopted (DerSimonian and Laird, 1986) [17]. Dose-response analysis was also conducted and $P<0.05$ was considered statistically significant. Cochran's Q-test and $I^{2}$ statistics were used to assess the 
heterogeneity of individual studies. $I^{2}>50 \%$ and $P<0.1$ were considered as statistically significant heterogeneity [18]. Fixed-effects model and random-effects model were adopted as the pooling method. We used the random-effects model to calculate the pooled RRs and 95 CIs when the evidence of heterogeneity was present $(>50 \%)$. Otherwise, if there was no obvious heterogeneity $(<50 \%)$, the fixed-effects model was used. Subgroup analyses were performed to explore the source of heterogeneity in this study.

The subgroup analyses were performed according to study design, geographic location, patient sex, number of cases, size of adenoma, type of colorectal lesion, study quality, and type of food frequency questionnaire (FFQ) with dairy consumption if data were permitted. Sensitivity analyses were conducted to examine the stability of results by omitting one study at a time. Publication bias was assessed with Begg's test and Egger's test if $\geq 10$ studies are available and it was considered to exist when $P<0.05$. The trim and fill method was used to reduce the potential influence of publication bias [19].

Moreover, if $\geq 3$ studies are available, a dose-response meta-analysis was carried out to estimate the trend between different exposure levels of dairy products and precursors of CRC using a random-effects meta-regression. Generalized least-squares trend (GLST) estimation modeling and spline curve modeling (MKspline STATA command) were used to estimate the dose-response relation analysis. Both linear and nonlinear dose-response analyses were performed with data from the included studies. The GLST and variance-weighted least-squares methods require median values for categories of intake levels. When medians and means were not presented, the category mid-point was used. If the dairy consumption was reported in servings/day, we converted it into grams/day as the following standard units: $200 \mathrm{~g}$ for total dairy, $200 \mathrm{~g}$ for milk (1 glass), $125 \mathrm{~g}$ for yogurt (1 cup), and $30 \mathrm{~g}$ for cheese [20]. If the cheese consumption was given in slices/day, we considered each slice as $25 \mathrm{~g}$ [21]. The doseresponse results were presented for a 200, 200, 50, and $25 \mathrm{~g} /$ day increment for total dairy products, total milk, yogurt, and cheese, respectively. All statistical analyses were performed using STATA, version 12.0 (Stata Corp., College Station, TX, USA).

\section{Results}

3.1. Search Results and Study Characteristics. Figure 1 shows the flow diagram of the identification and selection process of the included studies. Of the 1377 potentially relevant articles which initially retrieved, 563 duplicate articles were excluded and 58 articles remained for full-text review after screening the title and abstract. Among them, 46 articles were excluded (16 were review/meta-analysis/guideline articles, 14 did not report relative risk or sufficient data to calculate relative risk, 6 were abstracts and original articles were not available, 5 did not modify risk factor to different dairy products such as yogurt or cheese, 4 reported duplicated analysis from the same data source, and 1 did not report separately results of colorectal adenoma/cancer). In the end, a total of 12 eligible articles were included in this systematic review and meta-analysis: 9 case-control and 3 cohort studies.

The 12 included studies, published between 1991 and 2020, had 19957 cases with precursors of CRC. Of the studies, 6 were conducted in Europe, 4 in the United States, 1 in Iran, and 1 in Australia. Tables 1 and 2 show the main characteristics of the included studies.

3.2. Quality Assessment. All 12 studies were assessed to have a moderate risk of bias. Table S2 showed the study quality and bias risk of each domain of the included studies.

3.3. Highest Consumption Compared with Lowest Consumption Analysis. As shown in Table 3, a total of 12 studies assessed the effect of dairy consumption on the likelihood of colorectal adenomas and serrated lesions, which include 3 cohort studies [22-24] (15124 cases) and 9 case-control study [25-33] (4833 cases).

3.3.1. Total Dairy. A total of 5 studies assessed the effect of total dairy consumption on the likelihood of colorectal adenomas and serrated lesions, which include 1 cohort study [23] (516 cases) and 4 case-control studies [26, 27, 32, 33] (1862 cases). A significantly negative association of total dairy intake with colorectal adenomas and serrated lesions was observed among overall studies ( $R R=0.8095 \%$ CI: $0.69-0.93, P=0.003 ; I^{2}=4.6 \%$ ) (Figure 2). Evidence in favor of the association was weaker among cohort studies ( $\mathrm{RR}=0.80$ 95\% CI: $0.61-1.04, P=0.097)$ when compared with case-control studies ( $\mathrm{RR}=0.8095 \% \mathrm{CI}$ : 0.68-0.96, $\left.P=0.013 ; I^{2}=28.4 \%\right)$.

3.3.2. Total Milk. A total of 6 studies assessed the effect of total milk consumption on the likelihood of colorectal adenomas and serrated lesions, which included 2 cohort studies [22, 23] (1197 cases) and 4 case-control studies $[25,29,31,32]$ (1560 cases). The pooled summary effect size indicated no significant association in overall $(\mathrm{RR}=1.00$ 95\% CI: $\left.0.88-1.13, P=0.983 ; I^{2}=32.4 \%\right)$, cohort $(\mathrm{RR}=0.98$ 95\% CI: $\left.0.83-1.15, P=0.782 ; I^{2}=0 \%\right)$, and case-control $\left(\mathrm{RR}=1.03\right.$ 95\% CI: $\left.0.85-1.24, P=0.983 ; I^{2}=56.9 \%\right)$ studies (Figure 3).

3.3.3. Non/Low-Fat Milk. A total of 3 studies assessed the effect of non/low-fat milk consumption on the likelihood of colorectal adenomas and serrated lesions, which include 1 cohort study [22] (681 cases) and 2 case-control studies [25, 32] (1149 cases). No significant association between non/low-fat milk intake with colorectal adenomas and serrated lesions was observed among overall studies $\left(\mathrm{RR}=0.9695 \% \mathrm{CI}: 0.81-1.14, P=0.659 ; I^{2}=0 \%\right)$, cohort studies $(\mathrm{RR}=0.9895 \% \mathrm{CI}: 0.75-1.28, P=0.880)$, and case-control studies $(\mathrm{RR}=0.9595 \% \mathrm{CI}: 0.75-1.19$, $\left.P=0.649 ; I^{2}=0 \%\right)$. 


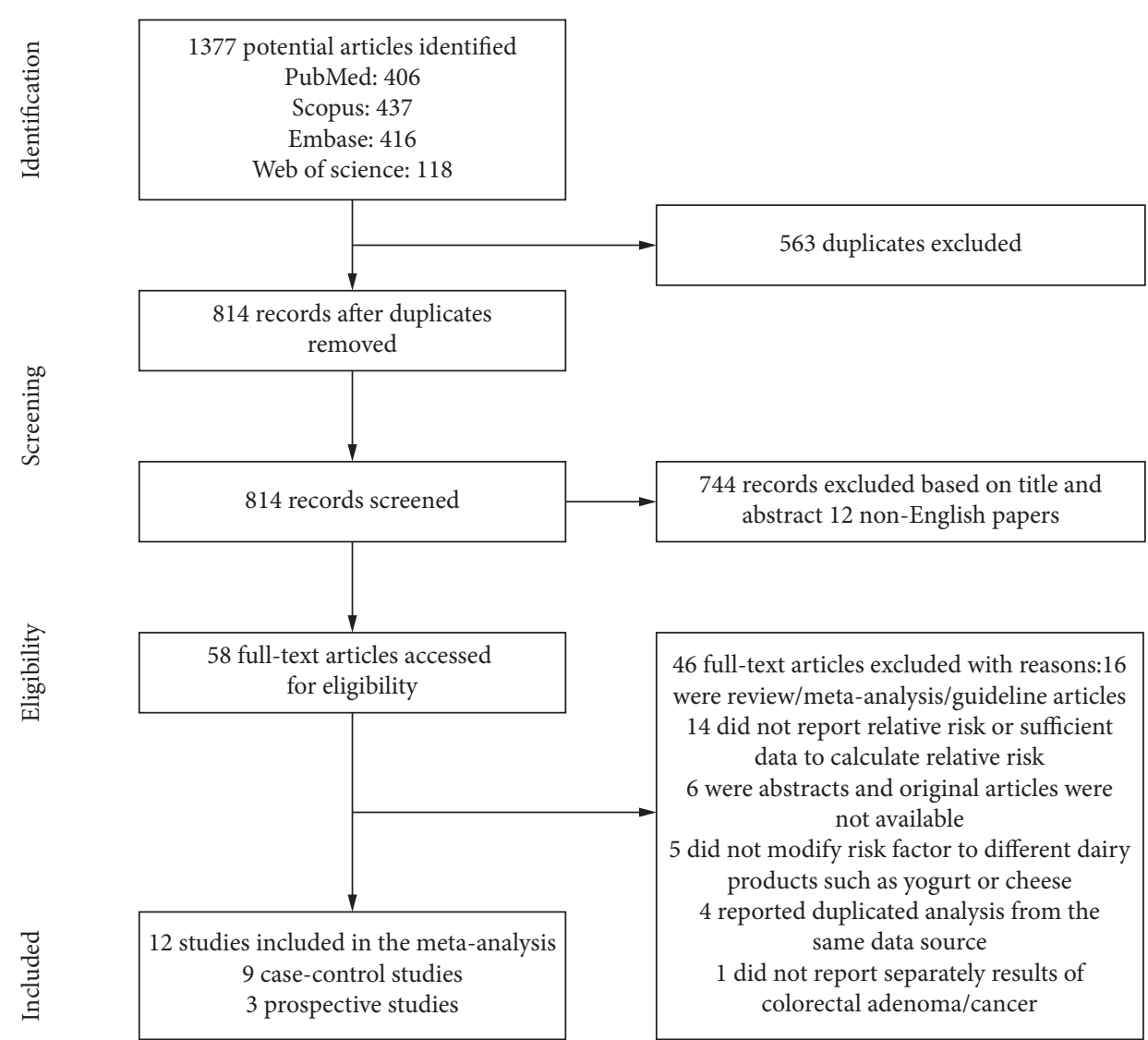

Figure 1: Flow of information through the different phases of the identification and selection of relevant studies examining the association between dairy consumption and the risk of the precursors of CRC.

3.3.4. Fermented Dairy Products. A total of 7 studies assessed the effect of fermented dairy product consumption on the likelihood of colorectal adenomas and serrated lesions, which included 3 cohort studies [22-24] (15124 cases) and 4 casecontrol studies [25, 28, 30, 31] (2922 cases). A significantly negative association of fermented dairy products intake with colorectal adenomas and serrated lesions was observed among overall studies ( $\mathrm{RR}=0.9795 \% \mathrm{CI}$ : 0.96-0.99, $P \leq 0.001$; $\left.I^{2}=41.9 \%\right)$, cohort studies $(\mathrm{RR}=0.92$ 95\%CI: $0.87-0.97$, $\left.P=0.002 ; I^{2}=0 \%\right)$, and case-control studies (RR $=0.9895 \%$ CI: $0.96-0.99, P=0.005 ; I^{2}=37.7 \%$ ) (Figure 4 ).

3.3.5. Yogurt. A total of 6 studies assessed the effect of yogurt consumption on the likelihood of colorectal adenomas and serrated lesions, which include 2 cohort studies [23, 24] (14443 cases) and 4 case-control studies [25, 28, 30, 31] $(2922$ cases). A negative association of yogurt intake with colorectal adenomas and serrated lesions was observed among overall studies ( $R R=0.93$ 95\% CI: $0.87-0.99, P=0.029 ; I^{2}=50.2 \%$ ). Evidence in favor of the association was weaker among casecontrol studies ( $\mathrm{RR}=0.93$ 95\% CI: $0.83-1.04, P=0.218$; $\left.I^{2}=24.9 \%\right)$ when compared with cohort studies $(\mathrm{RR}=0.91$ 95\%CI: $0.86-0.96, P \leq 0.001 ; I^{2}=0 \%$ ) (Figure 5).

3.3.6. Cheese. A total of 5 studies assessed the effect of cheese consumption on the likelihood of colorectal adenomas and serrated lesions, which include 2 cohort studies [22, 23] (1197 cases) and 3 case-control studies [25, 28, 31] (776 cases). Cheese intake was negatively associated with colorectal adenomas and serrated lesions among overall studies $\left(\mathrm{RR}=0.9695 \% \mathrm{CI}: 0.93-0.99, P=0.017 ; I^{2}=0 \%\right)$ and casecontrol studies $(\mathrm{RR}=0.96$ 95\%CI: $0.93-0.99, P=0.016$; $\left.I^{2}=0 \%\right)$. However, the results from the cohort study showed no significant association $(\mathrm{RR}=0.99$ 95\%CI: 0.81-1.22, $P=0.940 ; I^{2}=26.6 \%$ ) (Figure 6).

3.4. Dose-Response Meta-Analysis. Both linear and nonlinear dose-response analyses were performed. The potential nonlinear association was examined using restricted cubic splines with 4 knots fixed at the $5^{\text {th }}, 35^{\text {th }}, 65^{\text {th }}$, and $95^{\text {th }}$ percentiles of the distribution. Combining data from 3 studies $[26,30,33]$, trend meta-analysis showed a significant negative dose-response relationship in total dairy $\left(P_{\text {-nonlinearity }}=0.947\right)$ and yogurt $\quad\left(P_{\text {-nonlinearity }}=0.794\right)$ consumption from linearity. We found that $200 \mathrm{~g} / \mathrm{d}$ increment in total dairy consumption could decrease $12 \%$ risk of colorectal adenomas and serrated lesions using the fixedeffect model with no heterogeneity $(\mathrm{RR}=0.88,95 \% \mathrm{CI}$ : 0.81-0.95, $P=0.001 ; P_{h}=0.658$ ) (Figure $7(\mathrm{a})$ ). Further, we found the risk of colorectal adenomas and serrated lesions decreased by $8 \%$ with an increment of $50 \mathrm{~g}$ yogurt using the fixed-effect model (RR $=0.92,95 \% \mathrm{CI}$ : 0.85-0.99, $P=0.037$; $P_{h}=0.367$ ) (Figure 7(b)). 


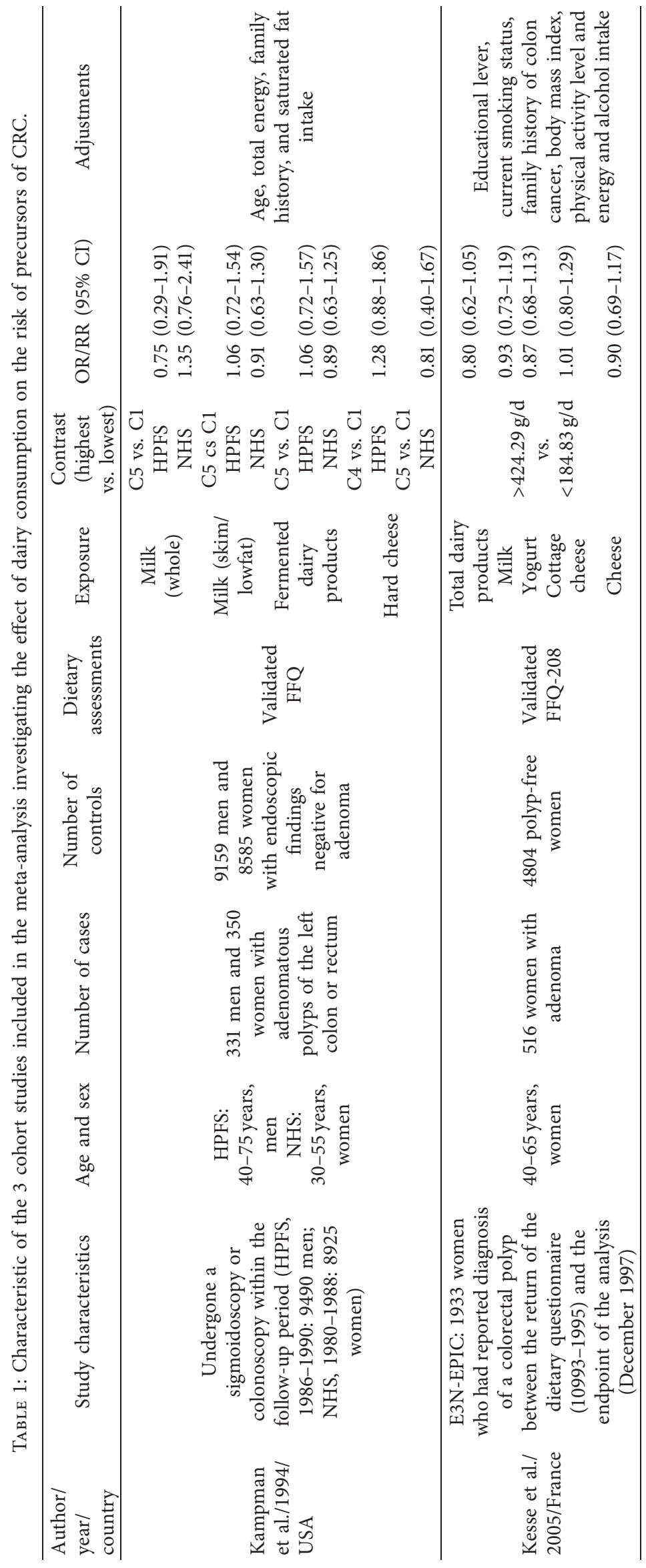




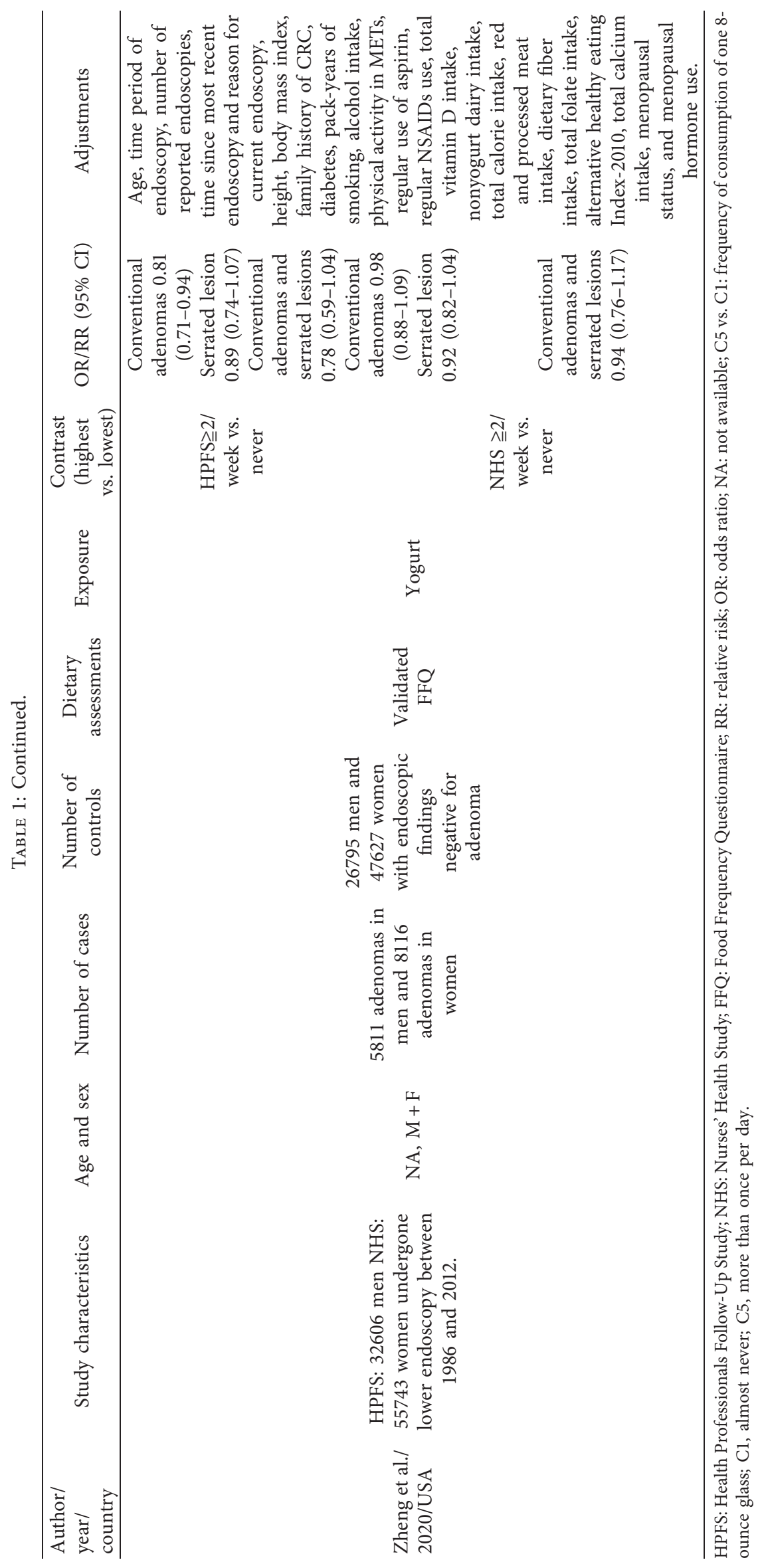


TABLE 2: Characteristic of the 9 case-control studies included in the meta-analysis investigating the effect of dairy consumption on the risk of precursors of CRC.

\begin{tabular}{|c|c|c|c|c|c|c|c|c|}
\hline $\begin{array}{l}\text { Author/ } \\
\text { year/ } \\
\text { country }\end{array}$ & Age and sex & $\begin{array}{l}\text { Number of } \\
\text { cases }\end{array}$ & $\begin{array}{l}\text { Number of } \\
\text { controls }\end{array}$ & $\begin{array}{c}\text { Dietary } \\
\text { assessments }\end{array}$ & Exposure & $\begin{array}{c}\text { Contrast } \\
\text { (highest vs. } \\
\text { lowest) }\end{array}$ & $\begin{array}{l}\text { OR }(95 \% \\
\text { CI })\end{array}$ & Adjustments \\
\hline \multirow{6}{*}{$\begin{array}{l}\text { Boutron } \\
\text { et al./1996/ } \\
\text { French }\end{array}$} & \multirow{6}{*}{$\begin{array}{l}\text { Aged 30-75, } \\
\qquad \mathrm{M}+\mathrm{F}\end{array}$} & \multirow[t]{3}{*}{$\begin{array}{l}154 \text { small } \\
\text { adenomas } \\
(<10 \mathrm{~mm})\end{array}$} & \multirow[t]{3}{*}{$\begin{array}{l}426 \text { polyp- } \\
\text { free }\end{array}$} & \multirow{6}{*}{$\begin{array}{l}\text { Validated } \\
\text { FFQ }\end{array}$} & $\begin{array}{l}\text { Total milk } \\
\text { Low-fat } \\
\text { milk } \\
\text { Hard and } \\
\text { semihard } \\
\text { cheese }\end{array}$ & Q5 vs. Q1 & $\begin{array}{c}1.1 \\
(0.6-1.9) \\
1.0 \\
(0.5-1.7) \\
1.2 \\
(0.6-2.4)\end{array}$ & \multirow{6}{*}{$\begin{array}{c}\text { Age, sex, and caloric } \\
\text { intake }\end{array}$} \\
\hline & & & & & $\begin{array}{l}\text { Cottage } \\
\text { cheese } \\
\text { Yogurt }\end{array}$ & Q3 vs. Q1 & $\begin{array}{c}1.0 \\
(0.7-1.6) \\
1.3 \\
(0.8-2.2)\end{array}$ & \\
\hline & & & & & Total milk & \multirow{3}{*}{ Q5 vs. Q1 } & $\begin{array}{c}1.0 \\
(0.5-2.0)\end{array}$ & \\
\hline & & \multirow{3}{*}{$\begin{array}{l}208 \text { large } \\
\text { adenomas } \\
(>10 \mathrm{~mm})\end{array}$} & \multirow{3}{*}{$\begin{array}{l}154 \text { small } \\
\text { adenomas } \\
(<10 \mathrm{~mm})\end{array}$} & & $\begin{array}{l}\text { Low-fat } \\
\text { milk }\end{array}$ & & $\begin{array}{c}1.1 \\
(0.5-2.1)\end{array}$ & \\
\hline & & & & & $\begin{array}{c}\text { Hard and } \\
\text { semihard } \\
\text { cheese }\end{array}$ & & $\begin{array}{c}1.1 \\
(0.5-2.5)\end{array}$ & \\
\hline & & & & & $\begin{array}{l}\text { Cottage } \\
\text { cheese } \\
\text { Yogurt }\end{array}$ & Q3 vs. Q1 & $\begin{array}{c}1.1 \\
(0.7-1.8) \\
0.5 \\
(0.3-0.9) \\
\end{array}$ & \\
\hline $\begin{array}{l}\text { Diergaarde } \\
\text { et al./2005/ } \\
\text { Dutch } \\
\end{array}$ & $\begin{array}{l}\text { Aged } 18-75 \\
\quad M+F\end{array}$ & 278 CRA & $\begin{array}{l}414 \text { polyp- } \\
\text { free }\end{array}$ & $\begin{array}{l}\text { Validated } \\
\text { FFQ-178 }\end{array}$ & $\begin{array}{l}\text { Dairy } \\
\text { products }\end{array}$ & $\begin{array}{l}\geqq 495.0 \mathrm{~g} / \mathrm{d} \text { vs. } \\
\quad \leqq 238.9 \mathrm{~g} / \mathrm{d}\end{array}$ & $\begin{array}{c}0.7 \\
(0.4-1.0)\end{array}$ & $\begin{array}{l}\text { Age, gender, and total } \\
\text { energy intake }\end{array}$ \\
\hline $\begin{array}{l}\text { Nasab et al./ } \\
\text { 2020/Iran }\end{array}$ & $\begin{array}{c}\text { Cases: aged } \\
56.46 \pm 10.01, \\
\text { controls: aged } \\
55.08 \pm 9.45 \\
\mathrm{M}+\mathrm{F}\end{array}$ & 139 CRA & $\begin{array}{l}240 \text { hospital } \\
\text { control }\end{array}$ & $\begin{array}{l}\text { Validated } \\
\text { FFQ-148 }\end{array}$ & Dairy & High vs. low & $\begin{array}{c}0.92 \\
(0.57-1.48)\end{array}$ & $\begin{array}{c}\text { Energy, smoking, } \\
\text { physical activity, age, } \\
\text { calcium } \\
\text { supplementation, } \\
\text { history of diabetes and } \\
\text { hypertension, cooking } \\
\text { type, levels of salt } \\
\text { intake, family history } \\
\text { of cancer }\end{array}$ \\
\hline $\begin{array}{l}\text { Karagianni } \\
\text { et al./2010/ } \\
\text { Greece }\end{array}$ & $\begin{array}{c}\text { Cases: aged } \\
\text { 30-77, } \\
\text { controls: } \\
33-80, \mathrm{M}+\mathrm{F}\end{array}$ & $\begin{array}{c}52 \text { advanced } \\
\text { colorectal } \\
\text { polyps }\end{array}$ & $\begin{array}{l}52 \text { healthy } \\
\text { control }\end{array}$ & $\begin{array}{l}\text { Validated } \\
\text { FFQ }\end{array}$ & $\begin{array}{c}\text { Milk } \\
\text { Yogurt }\end{array}$ & $\begin{array}{l}\text { Logistic } \\
\text { regression }\end{array}$ & $\begin{array}{c}\text { NA } \\
0.98 \\
(0.97-1.00) \\
\\
0.96 \\
(0.93-1.00)\end{array}$ & $\begin{array}{l}\text { Age, sex, smoking, } \\
\text { physical activity, BMI, } \\
\text { waist circumference, } \\
\text { hypercholesterolemia, } \\
\text { alcohol, yogurt, } \\
\text { cheese, red meat, fish, } \\
\text { fruits, vegetables, and } \\
\text { garlic }\end{array}$ \\
\hline & & & & & & Logistic re & gression & Age, sex, vegetable, \\
\hline $\begin{array}{l}\text { Kune et al./ } \\
1991 / \\
\text { Australia }\end{array}$ & $\begin{array}{c}\text { Cases: aged } \\
68 \pm 9, \\
\text { controls: aged } \\
65 \pm 11, \mathrm{M}+\mathrm{F}\end{array}$ & $\begin{array}{l}49 \text { colorectal } \\
\text { adenomatous } \\
\text { polyps larger } \\
\text { than } 1 \mathrm{~cm}\end{array}$ & $\begin{array}{c}727 \\
\text { community } \\
\text { controls }\end{array}$ & $\begin{array}{l}\text { Validated } \\
\text { FFQ }\end{array}$ & $\begin{array}{c}\text { Milk } \\
\text { drinks }\end{array}$ & Female & $\begin{array}{c}3.70 \\
(1.44-9.52) \\
\\
1.50 \\
(0.50-4.54)\end{array}$ & $\begin{array}{l}\text { cruciferous vegetables, } \\
\text { vitamin C, beef, pork, } \\
\text { fish, fat, milk drinks, } \\
\text { vitamin supplement, } \\
\text { beer, family history of } \\
\text { CRC in near relatives }\end{array}$ \\
\hline
\end{tabular}


TABLe 2: Continued.

\begin{tabular}{|c|c|c|c|c|c|c|c|c|}
\hline $\begin{array}{l}\text { Author/ } \\
\text { year/ } \\
\text { country }\end{array}$ & Age and sex & $\begin{array}{l}\text { Number of } \\
\text { cases }\end{array}$ & $\begin{array}{l}\text { Number of } \\
\text { controls }\end{array}$ & $\begin{array}{c}\text { Dietary } \\
\text { assessments }\end{array}$ & Exposure & $\begin{array}{l}\text { Contrast } \\
\text { (highest vs. } \\
\text { lowest) } \\
\end{array}$ & $\begin{array}{l}\text { OR }(95 \% \\
\quad \mathrm{CI})\end{array}$ & Adjustments \\
\hline $\begin{array}{l}\text { Rifkin et al./ } \\
\text { 2020/USA }\end{array}$ & $\begin{array}{l}\text { TCPS: aged } \\
\text { 40-75, JHBS: } \\
\text { aged } 40-85 \\
\quad \mathrm{M}+\mathrm{F}\end{array}$ & $\begin{array}{c}\text { Colorectal } \\
\text { polyps TCPS: } \\
\text { 181 SSP, } 1536 \\
\text { AP JHBS: } 96 \\
\text { SSP, } 333 \text { AP }\end{array}$ & $\begin{array}{l}\text { Polyp-free } \\
\text { controls } \\
\text { TCPS: } 3258 \\
\text { JHBS: } 579\end{array}$ & $\begin{array}{l}\text { Validated } \\
\text { FFQ-108 }\end{array}$ & Yogurt & $\begin{array}{c}\text { Daily vs. } \\
\text { never } \\
\geqq 0.20 \text { cups vs. } \\
\text { none/rarely } \\
\text { JHB }\end{array}$ & $\begin{array}{l}\text { AP } 0.93 \\
(0.69-1.25) \\
\text { SSP } 0.49 \\
(0.19-1.24) \\
\text { AP } 0.98 \\
(0.79-1.22) \\
\text { SSP } 0.77 \\
(0.43-1.36) \\
\text { S AP } 0.75 \\
(0.54-1.04)\end{array}$ & $\begin{array}{c}\text { TCPS: sex, study } \\
\text { location, age, regular } \\
\text { alcohol drinking } \\
\text { status, BMI, smoking } \\
\text { status, physical activity } \\
\text { in the past } 10 \text { years, } \\
\text { educational } \\
\text { attainment, NSAID } \\
\text { use, red meat intake, } \\
\text { dietary energy intake, } \\
\text { and frequency of } \\
\text { nonyogurt dairy intake } \\
\text { HBS: sex, age, cigarette } \\
\text { use, overweight, prior } \\
\text { colon polyp, history of } \\
\text { cholecystectomy, } \\
\text { diabetes mellitus } \\
\text { diagnosis, } \\
\text { hypertension } \\
\text { diagnosis, } \\
\text { hyperlipidemia } \\
\text { diagnosis, physical } \\
\text { activity and }>10 \\
\text { alcohol drinks/week. }\end{array}$ \\
\hline $\begin{array}{l}\text { Senesse } \\
\text { et al./2009/ } \\
\text { France }\end{array}$ & $\begin{array}{l}\text { Aged 30-79, } \\
\quad M+F\end{array}$ & $\begin{array}{c}154 \text { small } \\
\text { adenomas } \\
(<10 \mathrm{~mm}), \\
208 \text { large } \\
\text { adenomas } \\
(\geq 10 \mathrm{~mm})\end{array}$ & $\begin{array}{l}427 \text { polyp- } \\
\text { free controls }\end{array}$ & $\begin{array}{l}\text { Validated } \\
\text { FFQ }\end{array}$ & Milk & Q4 vs. Q1 & $\begin{aligned} &< 10 \mathrm{~mm} 1.0 \\
&(0.6-1.7) \\
& \geqq 10 \mathrm{~mm} \\
& 1.0(0.7-1.7) \\
&<10 \mathrm{~mm} 1.2 \\
&(0.8-2.1) \\
& \geqq 10 \mathrm{~mm} 0.6 \\
&(0.4-1.0) \\
&<10 \mathrm{~mm} 1.0 \\
&(0.5-1.7) \\
& \geqq 10 \mathrm{~mm} \\
& 0.8(0.5-1.4)\end{aligned}$ & $\begin{array}{c}\text { Age, gender, energy } \\
\text { intake, body mass } \\
\text { index, alcohol, and } \\
\text { tobacco }\end{array}$ \\
\hline $\begin{array}{l}\text { Um et al./ } \\
\text { 2016/USA }\end{array}$ & $\begin{array}{l}\text { Aged } 35-74, \\
\qquad M+F\end{array}$ & 787 CRA & $\begin{array}{l}2033 \text { polyp- } \\
\text { free controls }\end{array}$ & $\begin{array}{l}\text { Validated } \\
\text { FFQ }\end{array}$ & $\begin{array}{c}\text { Total milk } \\
\text { products } \\
\text { Total milk } \\
\text { Whole } \\
\text { milk }\end{array}$ & Q5 vs. Q1 & $\begin{array}{c}0.99 \\
(0.74-1.34) \\
0.90 \\
(0.68-1.19) \\
1.15 \\
(0.89-1.49)\end{array}$ & $\begin{array}{l}\text { Study, age, sex, } \\
\text { oxidative balance } \\
\text { score, family history of } \\
\text { colorectal cancer in } \\
\text { first-degree relative, } \\
\text { regular use of aspirin } \\
\text { or nonsteroidal anti- } \\
\text { inflammatory drugs, } \\
\text { total energy intake, } \\
\text { total fat intake (energy } \\
\text { adjusted), } \\
\text { supplemental calcium } \\
\text { intake. }\end{array}$ \\
\hline $\begin{array}{l}\text { Wark et al./ } \\
\text { 2006/ } \\
\text { Netherlands }\end{array}$ & $\begin{array}{l}\text { Aged } 18-75 \\
\qquad M+F\end{array}$ & $\begin{array}{l}658 \text { CRA (81 } \\
\text { K-ras }^{+}, 453 \mathrm{~K}- \\
\text { Ras }^{-}, 124 \text { NA) }\end{array}$ & $\begin{array}{l}709 \text { polyp- } \\
\text { free controls }\end{array}$ & $\begin{array}{l}\text { Validated } \\
\text { FFQ-178 }\end{array}$ & $\begin{array}{l}\text { Dairy } \\
\text { products }\end{array}$ & $\begin{array}{c}>238.2-474.6 \\
\text { vs. } \leqq 238.2 \\
>474.6 \text { vs. } \\
>238.2-474.6\end{array}$ & $\begin{array}{c}0.89(0.67 \\
1.18) \\
0.91(0.68 \\
1.22)\end{array}$ & $\begin{array}{l}\text { Sex, age, and total } \\
\text { energy. }\end{array}$ \\
\hline
\end{tabular}

TCPS: Tennessee Colorectal Polyp Study; JHBS: Johns Hopkins Biofilm Study; FFQ: Food Frequency Questionnaire; AP: adenomatous polyp; SSP: sessile serrated polyp; OR: odds ratio; NA: not available; Q: quintiles. 
TABLE 3: Analysis of highest versus lowest dairy consumption and risk of colorectal adenomas and serrated lesions.

\begin{tabular}{|c|c|c|c|c|c|c|}
\hline & \multirow[b]{2}{*}{ Factors } & \multirow{2}{*}{ Number of studies } & \multirow{2}{*}{ Pooled RR (95\% CI) } & \multirow{2}{*}{$P$ value } & \multicolumn{2}{|c|}{ Heterogeneity } \\
\hline & & & & & $I^{2}(\%)$ & $p_{h}$ \\
\hline \multirow{3}{*}{ Total dairy } & Total & 5 & $0.80(0.69,0.93)$ & 0.003 & 4.6 & 0.381 \\
\hline & Cohort & 1 & $0.80(0.61,1.04)$ & 0.097 & - & - \\
\hline & Case-control & 4 & $0.80(0.68,0.96)$ & 0.013 & 28.4 & 0.241 \\
\hline \multirow{3}{*}{ Total milk } & Total & 6 & $1.00(0.88,1.13)$ & 0.983 & 32.4 & 0.193 \\
\hline & Cohort & 2 & $0.98(0.83,1.15)$ & 0.782 & 0 & 0.589 \\
\hline & Case-control & 4 & $1.03(0.85,1.24)$ & 0.983 & 56.9 & 0.073 \\
\hline \multirow{3}{*}{ Non/low-fat milk } & Total & 3 & $0.96(0.81,1.14)$ & 0.659 & 0 & 0.890 \\
\hline & Cohort & 1 & $0.98(0.75,1.28)$ & 0.880 & - & - \\
\hline & Case-control & 2 & $0.95(0.75,1.19)$ & 0.649 & 0 & 0.656 \\
\hline \multirow{3}{*}{ Fermented dairy products } & Total & 7 & $0.97(0.96,0.99)$ & $\leq 0.001$ & 41.9 & 0.111 \\
\hline & Cohort & 3 & $0.92(0.87,0.97)$ & 0.002 & 0 & 0.899 \\
\hline & Case-control & 4 & $0.98(0.96,0.99)$ & 0.005 & 37.7 & 0.186 \\
\hline \multirow{3}{*}{ Yogurt } & Total & 6 & $0.93(0.87,0.99)$ & 0.029 & 50.2 & 0.074 \\
\hline & Cohort & 2 & $0.91(0.86,0.96)$ & $\leq 0.001$ & 0 & 0.736 \\
\hline & Case-control & 4 & $0.93(0.83,1.04)$ & 0.218 & 24.9 & 0.262 \\
\hline \multirow{3}{*}{ Cheese } & Total & 5 & $0.96(0.93,0.99)$ & 0.017 & 0 & 0.711 \\
\hline & Cohort & 2 & $0.99(0.81,1.22)$ & 0.940 & 26.6 & 0.243 \\
\hline & Case-control & 3 & $0.96(0.93,0.99)$ & 0.016 & 0 & 0.713 \\
\hline
\end{tabular}

$P_{h:} P$ value for heterogeneity.

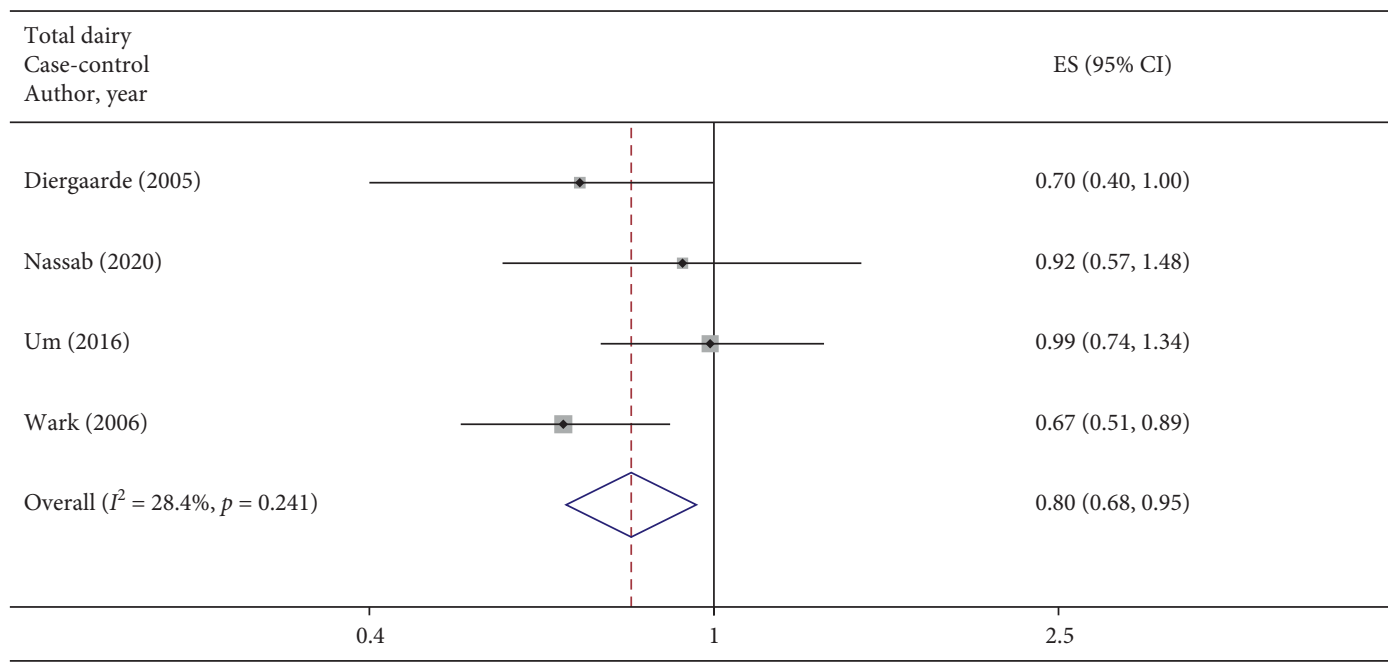

FIGURE 2: Fixed-effects meta-analysis of studies that examined total dairy consumption and risk of colorectal adenomas and serrated lesions. ES, effect size.

3.5. Subgroup and Sensitivity Analyses. The results of subgroup analyses conducted to explore the sources of heterogeneity were detailed shown in Table S3. Intake of total dairy, fermented dairy products, yogurt, and cheese was significantly associated with a decreased risk of colorectal adenomas and serrated lesions in most subgroup analysis. When stratified by sex, an inverse association was observed for intake of fermented dairy products in both men $(\mathrm{SRR}=0.85,95 \% \mathrm{CI}: 0.77-0.93)$ and women $(\mathrm{SRR}=0.92$, 95\% CI: 0.87-0.98). When stratified by the size of adenoma, an inverse association was observed for intake of yogurt and cheese in studies with a size of $10 \mathrm{~mm}$ or more $(\mathrm{SRR}=0.71 \quad 95 \% \quad \mathrm{CI}: \quad 0.51-0.99 ; \quad \mathrm{SRR}=0.96 \quad 95 \% \quad \mathrm{CI}$ :
0.93-0.99) but not among studies with a size of less than $10 \mathrm{~mm}(\mathrm{SRR}=1.0195 \% \mathrm{CI}: 0.81-1.25 ; \mathrm{SRR}=1.0895 \% \mathrm{CI}$ : $0.68-1.71)$. Intake of total milk was not significantly associated with the risk of colorectal adenomas and serrated lesions in subgroup analyses. Too few studies of non/lowfat milk precluded any meaningful subgroup analysis.

Sensitivity analyses were conducted to evaluate the influence of a single study on the overall risk estimate by omitting one study in each turn (Table S4). In the analysis of total milk, the heterogeneity decreased from $56.9 \%$ to $0 \%$ when omitting the study by Kune et al. In the analysis of yogurt, the heterogeneity decreased from $50.2 \%$ to $0 \%$ when omitting the study by Karagianni et al. 


\begin{tabular}{l} 
Total milk \\
Author, year \\
\hline Cohort \\
Kampman et al. (1994) \\
Kesse et al. (2005) \\
Subtotal $\left(I^{2}=0.0 \%, p=0.589\right)$ \\
Case-control \\
Boutron et al. (1996) \\
Kune et al. (1991) \\
Senesse et al. (2002) \\
Um et al. $(2016)$ \\
Subtotal $\left(I^{2}=56.9 \%, p=0.073\right)$ \\
Heterogeneity between groups: $p=0.698$ \\
Overall $\left(I^{2}=32.4 \%, p=0.193\right)$
\end{tabular}

FIGURE 3: Fixed-effects meta-analysis of studies that examined total milk consumption and risk of colorectal adenomas and serrated lesions. ES, effect size.

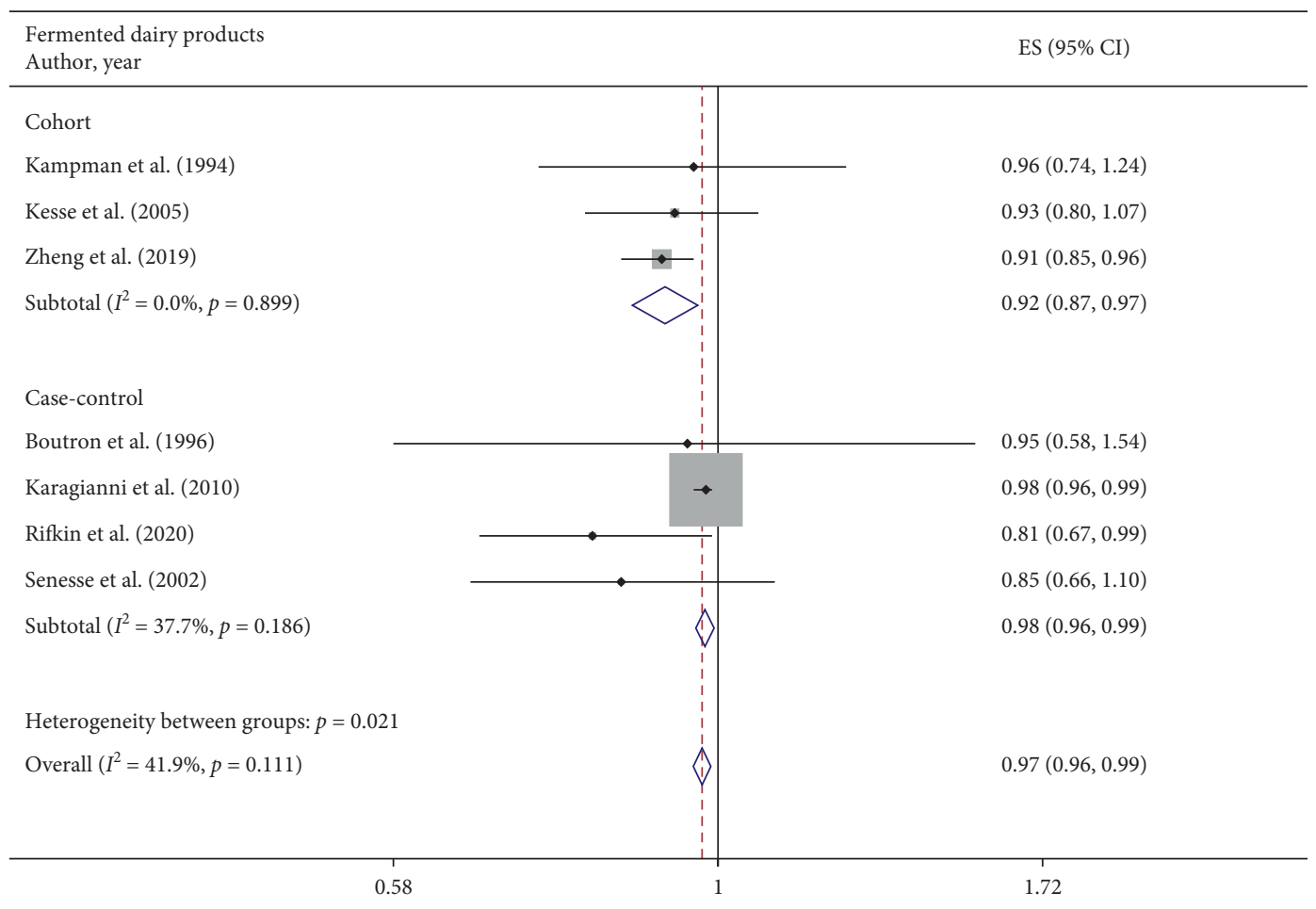

FIGURE 4: Fixed-effects meta-analysis of studies that examined fermented dairy products consumption and risk of colorectal adenomas and serrated lesions. ES, effect size. 


\begin{tabular}{|c|c|}
\hline $\begin{array}{l}\text { Yogurt } \\
\text { Author, year }\end{array}$ & ES $(95 \%$ CI $)$ \\
\hline \multicolumn{2}{|l|}{ Cohort } \\
\hline Kesse et al. (2005) & $0.87(0.68,1.13)$ \\
\hline Zheng et al. (2019) & $0.91(0.85,0.96)$ \\
\hline Subtotal $\left(I^{2}=0.0 \%, p=0.736\right)$ & $0.91(0.86,0.96)$ \\
\hline \multicolumn{2}{|l|}{ Case-control } \\
\hline \multicolumn{2}{|l|}{ Boutron et al. (1996) } \\
\hline Karagianni et al. (2010) & $0.98(0.97,1.00)$ \\
\hline Rifkin et al. (2020) & $0.81(0.67,0.99)$ \\
\hline \multicolumn{2}{|l|}{ Senesse et al. (2002) } \\
\hline Subtotal $\left(I^{2}=24.9 \%, p=0.262\right)$ & $0.93(0.83,1.04)$ \\
\hline Overall $\left(I^{2}=50.2 \%, p=0.074\right)$ & $0.93(0.87,0.99)$ \\
\hline Note: weights are from random effects analysis & \\
\hline 0.32 & 3.13 \\
\hline
\end{tabular}

FIGURE 5: Fixed-effects meta-analysis of studies that examined yogurt consumption and risk of colorectal adenomas and serrated lesions. ES, effect size.

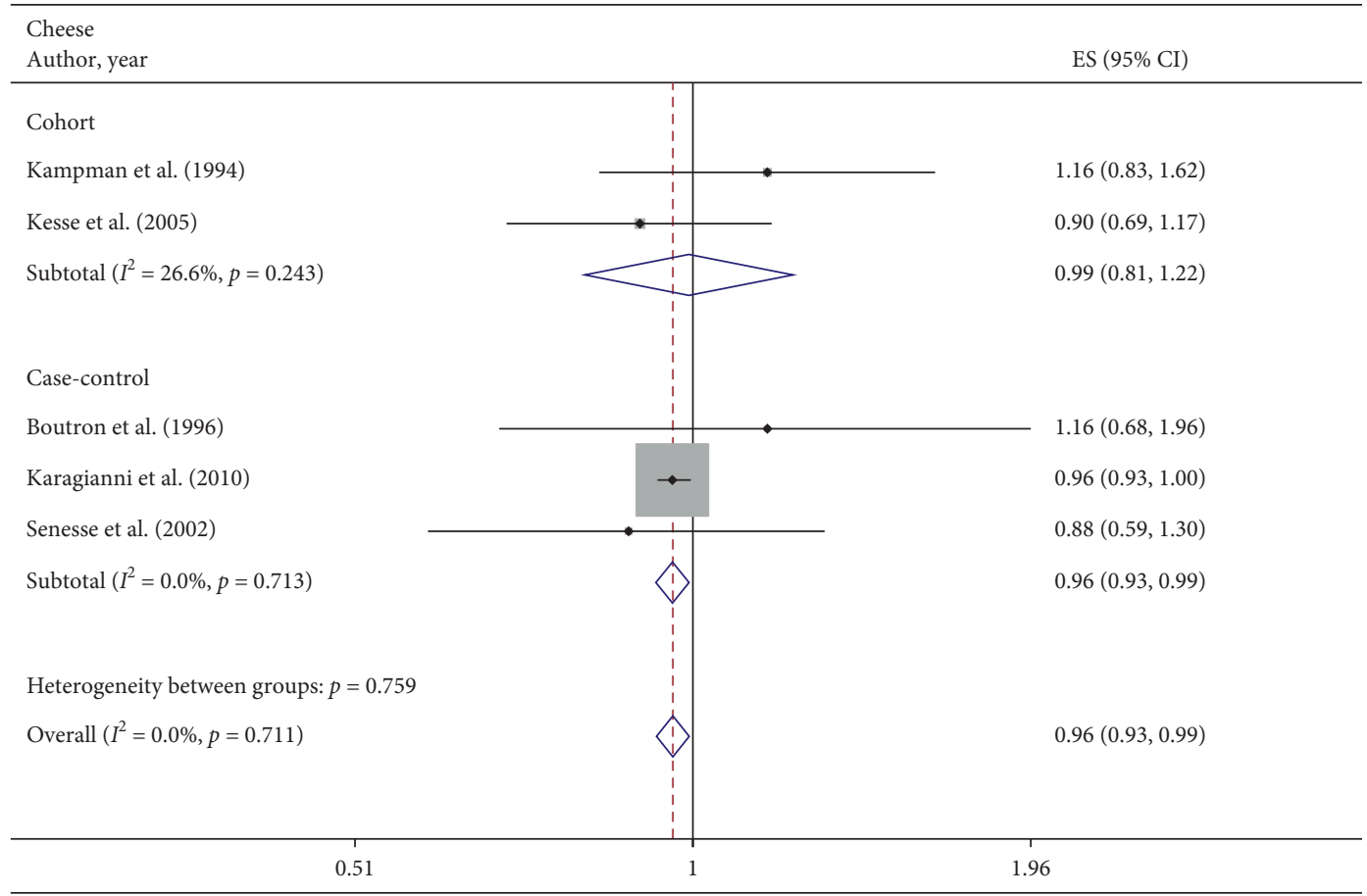

FIGURE 6: Fixed-effects meta-analysis of studies that examined cheese consumption and risk of colorectal adenomas and serrated lesions. ES, effect size.

\section{Discussion}

By pooling the 12 observational studies ( 9 case-control and 3 cohort studies) eventually included, this present metaanalysis was the first study that conclusively indicated an inverse association between fermented dairy products including yogurt and cheese and the risk of conventional adenomas and serrated lesions, of which fermented dairy products intake showed a decreased trend in both cohort and case-control studies but cheese and yogurt consumption 


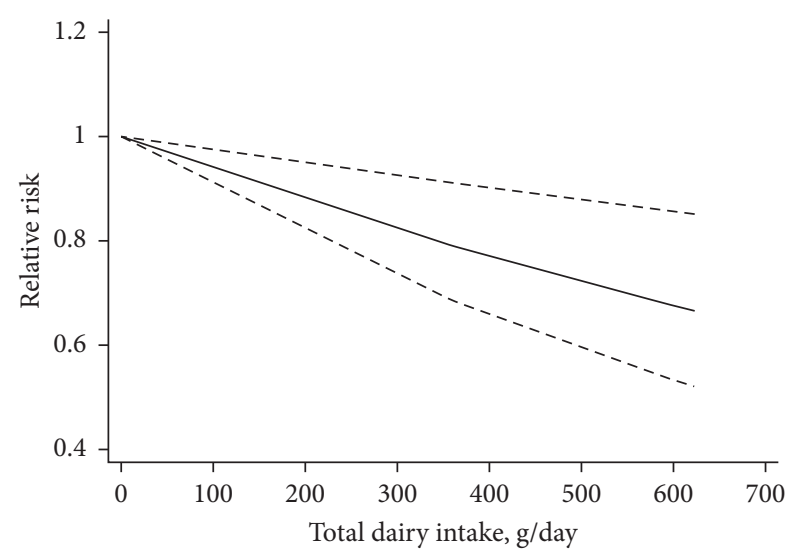

(a)

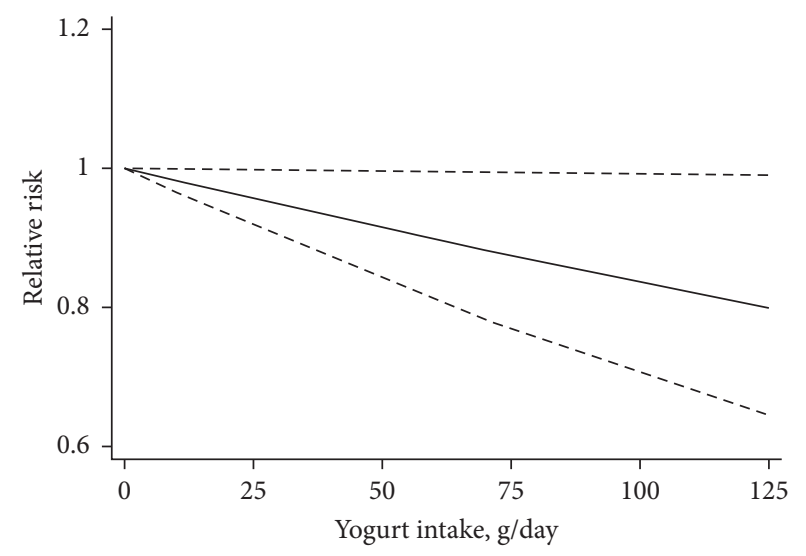

(b)

FiguRe 7: The Linear dose-response meta-analysis of total dairy (a) and yogurt (b) intake with relative risk of colorectal adenomas and serrated lesions. Weights are from the fixed-effects analysis. Solid line represents the linear trend. Lines with short dashes represent the $95 \%$ CI.

display an inverse association only in case-control studies or cohort studies. No significant associations were found for the consumption of total milk and non/low-fat milk. Very few studies have evaluated the association between whole milk and cottage cheese with the risk of conventional adenomas and serrated lesions. The negative associations of total dairy, yogurt, and cheese were not supported by cohort studies which may be explained by many reasons such as the differences in study design, study quality, category of the intake frequency, amounts of dairy intake, and potential confounders.

CRC is arising through three major pathways, including adenoma-carcinoma sequence, serrated pathway, and inflammatory pathway [34]. It was estimated that approximately $10-15 \%$ of the sporadic CRC were progressed from a serrated polyp and 85-90\% were progressed from adenomatous polyps. The subset of serrated polyps includes HP (hyperplastic polyp), TSA, and SSA/P [35]. HP is the most prevalent type of serrated polyps which is the lack of malignant potential [36]. Therefore, we excluded the data investigating risk between dairy and HP. No more than $10 \%$ of the adenomas will progress to CRC. Compared with nonadvanced adenomas, advanced adenomas $(\geq 10 \mathrm{~mm}$ in diameter, villous histology, or high-grade dysplasia, with or without $>3$ adenomas) are more likely to develop into malignancy [35]. Because high-risk adenoma is more likely to develop into malignancy than low-risk adenoma, the data of high-risk adenoma are more informative for preventive strategies. The result of this meta-analysis showed a negative association between yogurt and cheese intake and adenoma $\geq 10 \mathrm{~mm}$, suggesting that fermented dairy products may play a protective role against the progression of CRC. However, the result should be interpreted with caution because only a few studies were involved in this meta-analysis.

Dairy foods may decrease the risk of precursors of CRC through several mechanisms. Dairy foods are the main source of calcium in the diet. In the most recent report from the WCRF/AICR, low calcium intake may increase CRC risk. A previous observational study has shown a negative association between supplemental calcium intake and CRC risk [37]. On the one hand, calcium can decrease cell proliferation induced [38].

On the other hand, calcium can have an impact on several intracellular pathways leading to apoptosis in transformed cells and differentiation in normal cells $[39,40]$. The results of RCT showed that, in normal appearing colorectal mucosa of individuals with a history of adenoma, the expression of APC (adenomatous polyposis coli) and $\beta$-catenin is modified by calcium supplementation [41, 42]. Moreover, besides calcium, vitamin D, and folate are also micronutrients which might have protective effects [43].

We found that total dairy and fermented dairy products were inversely associated with the risk of the precursors of CRC while total milk was not associated with the risk. There are several mechanisms proposed to explain how fermented dairy food decreases the risk of precursors of CRC. Since the process of fermentation, fermented dairy foods contain plentiful probiotic such as Lactobacillus and Bifidobacterium [44-46]. The microbiota in the human body can form a microenvironment to alter cancer susceptibility and progression [47]. In a case-control study, CRC-associated microbiota was found to change with the degree of malignancy along the adenoma-carcinoma sequence [48]. According to this evidence, the gut microbiome, which can correct microbiota composition, modulate the innate immune system, restore gut barrier function, prevent pathogen colonization, and exert selective cytotoxicity against tumor cells, plays an important role in the development of CRC [49]. Yogurt has been recommended by the Dietary Guideline for Americans [50]. Live microorganisms such as Streptococcus thermophilus and Lactobacillus delbrueckii subsp. bulgaricus, two lactic acid bacteria used to ferment, may prevent carcinogenesis from initiating [51]. Various studies also have reported a protective effect of probiotics or prebiotics in CRC mice models as reviewed [52]. Furthermore, yogurt has been suggested to induce gastrointestinal hormone secretion [53, 54]. 
Our systematic review and meta-analysis had several strengths. Most of the current meta-analysis focus on the relationship between dietary factors such as milk or dairy products and the risk of CRC. We conducted the first metaanalysis of the association between dairy consumption and the risk of precursors of CRC including the conventional and serrated lesions, based on highest versus lowest analysis, linear and nonlinear dose-response meta-analysis. We included both cohort and case-control studies through a systematic search. All of the included studies used a validated FFQ to assess dairy consumption. Further, a significantly inverse dose-response relationship was observed between total dairy and yogurt consumption and the risk of precursors of CRC, which may strengthen the reliability of the results of our hypothesis. We also carried out a sensitivity analysis to investigate whether a particular study could explain the results.

However, our meta-analysis had several limitations. The main limitation of the study was a small number of included studies and subjects, so further subgroup analyses were not able to perform according to anatomical location and histology type. Dose-response analyses of specific types of dairy products were also limited because a few studies reported the modifiable risk factor with 95\% CI to different quantitative categories of exposure to dairy products. Secondly, the observed inverse association between dairy intake and precursors of CRC may be due to unmeasured or potential residual confounding, although the quality of 12 included studies was evaluated to have a moderate risk of bias by ROBINS-I tool. Although some known confounding factors were adjusted in most of the studies, not all potential confounders were adjusted for in every study. We found that the association between total dairy, fermented dairy products, yogurt, and cheese persisted in most subgroups, with adjustment for confounding factors. Thirdly, significant heterogeneity was observed among studies. However, there was no evidence of significant heterogeneity found between subgroups analyses. Fourthly, most of the case-control studies may not avoid recall and selection bias, especially dietary recall bias. Lastly, potential publication bias might have influenced the results.

\section{Conclusion}

This systematic review and meta-analysis was the first to assess the relationship between different dairy products and the precursor of CRC risk as well as the first to explore a linear association between them. In conclusion, our study suggests an inverse relationship between total dairy, fermented dairy products, yogurt, cheese, and risk of precursors of CRC, though the evidence was limited. However, no harmful effects were found between the intake of total milk and the risk of the precursor of CRC. Knowledge of risk factors associated with the precursors of CRC is important in prevention strategies. More large and precise prospective studies, as well as clinical trials, are needed to further investigate the associations and mechanisms between them. Future studies should also focus on the interactions between gut microbiota and environmental factors and their influences on colorectal carcinogenesis.

\section{Data Availability}

No data were used to support this study.

\section{Conflicts of Interest}

The authors declare that they have no conflicts of interest.

\section{Authors' Contributions}

Liliangzi Guo, M.D., Yuting Li, M.D., Jun Yao, Ph.D., and Lisheng Wang, Ph.D., contributed equally to this work. L.L.Z.G, Y.T.L, J.Y, and L.S.W were responsible for the design of the study and reviewed the manuscript. S.H.T screened the articles. Y.T.L and W.W.C abstracted the data. K.Y.H and L.X assessed the methodological quality of the studies. L.L.Z.G and Y.T.L performed statistical analysis and drafted the manuscript. S.H.T was responsible for revising the manuscript. All authors have read and approved the final manuscript.

\section{Supplementary Materials}

Supplemental Table 1. Definition of dairy products as described in the paper of 12 studies included in the metaanalysis. Supplementary Table 2. Bias risk of each domain of included studies assessed by ROBINS-I. Supplemental Table 3. Subgroup analyses of total dairy, fermented dairy products, total milk, yogurt, and cheese and the risk of colorectal adenomas and serrated lesions. Supplemental Table 4 . The sensitivity analysis of the relationship between total milk intake and risk of colorectal adenomas and serrated lesions (Supplementary Materials)

\section{References}

[1] F. Bray, J. Ferlay, I. Soerjomataram, R. L. Siegel, L. A. Torre, and A. Jemal, "Global cancer statistics 2018: GLOBOCAN estimates of incidence and mortality worldwide for 36 cancers in 185 countries," CA: A Cancer Journal for Clinicians, vol. 68, no. 6, pp. 394-424, 2018.

[2] J.-L. Wang, C.-H. Chang, J.-W. Lin, L.-C. Wu, L.-M. Chuang, and M.-S. Lai, "Infection, antibiotic therapy and risk of colorectal cancer: a nationwide nested case-control study in patients with type 2 diabetes mellitus," International Journal of Cancer, vol. 135, no. 4, pp. 956-967, 2014.

[3] Y.-L. Feng, L. Shu, P.-F. Zheng et al., "Dietary patterns and colorectal cancer risk: a meta-analysis," European Journal of Cancer Prevention, vol. 26, no. 3, pp. 201-211, 2017.

[4] L. Schwingshackl and G. Hoffmann, "Adherence to Mediterranean diet and risk of cancer: an updated systematic review and meta-analysis of observational studies," Cancer Medicine, vol. 4, no. 12, pp. 1933-1947, 2015.

[5] J. Godos, M. Tieri, F. Ghelfi et al., "Dairy foods and health: an umbrella review of observational studies," International Journal of Food Sciences and Nutrition, vol. 71, no. 2, pp. 138-151, 2020.

[6] S. K. Clinton, E. L. Giovannucci, and S. D. Hursting, "The World cancer research fund/American Institute for cancer research third expert report on diet, nutrition, physical activity, and cancer: impact and future directions," The Journal of Nutrition, vol. 150, no. 4, pp. 663-671, 2020. 
[7] X. Xu, E. Yu, X. Gao et al., "Red and processed meat intake and risk of colorectal adenomas: a meta-analysis of observational studies," International Journal of Cancer, vol. 132, no. 2, pp. 437-448, 2013.

[8] D. Aune, D. S. M. Chan, A. R. Vieira et al., "Red and processed meat intake and risk of colorectal adenomas: a systematic review and meta-analysis of epidemiological studies," Cancer Causes \& Control, vol. 24, no. 4, pp. 611-627, 2013.

[9] Q. Ben, Y. Sun, R. Chai, A. Qian, B. Xu, and Y. Yuan, "Dietary fiber intake reduces risk for colorectal adenoma: a metaanalysis," Gastroenterology, vol. 146, no. 3, pp. 689-699, 2014.

[10] N. Keum, D. H. Lee, D. C. Greenwood, X. Zhang, and E. L. Giovannucci, "Calcium intake and colorectal adenoma risk: dose-response meta-analysis of prospective observational studies," International Journal of Cancer, vol. 136, no. 7, pp. 1680-1687, 2015.

[11] P. A. Wark, R. Lau, T. Norat, and E. Kampman, "Magnesium intake and colorectal tumor risk: a case-control study and meta-analysis," The American Journal of Clinical Nutrition, vol. 96, no. 3, pp. 622-631, 2012.

[12] Y. Ou, B. Jiang, X. Wang, W. Ma, and J. Guo, "Selenium and colorectal adenomas risk: a meta-analysis," Nutrition and Cancer, vol. 64, no. 8, pp. 1153-1159, 2012.

[13] Y. Wang, J. Chen, R. Zhao et al., "Dose-response metaanalysis of coffee consumption and risk of colorectal adenoma," European Journal of Clinical Nutrition, vol. 74, no. 2, pp. 297-306, 2020.

[14] B. Xu, J. Sun, Y. Sun, L. Huang, Y. Tang, and Y. Yuan, "No evidence of decreased risk of colorectal adenomas with white meat, poultry, and fish intake: a meta-analysis of observational studies," Annals of Epidemiology, vol. 23, no. 4, pp. 215-222, 2013.

[15] D. Moher, A. Liberati, J. Tetzlaff, and D. G. Altman, "Preferred reporting items for systematic reviews and meta-analyses: the PRISMA statement," BMJ, vol. 339, p. b2535, 2009.

[16] J. A. Sterne, M. A. Hernán, B. C. Reeves et al., "ROBINS-I: a tool for assessing risk of bias in non-randomised studies of interventions," BMJ, vol. 355, p. i4919, 2016.

[17] R. DerSimonian and N. Laird, "Meta-analysis in clinical trials," Controlled Clinical Trials, vol. 7, no. 3, pp. 177-188, 1986.

[18] B. Chen and A. Benedetti, "Quantifying heterogeneity in individual participant data meta-analysis with binary outcomes," Systematic Review, vol. 6, no. 1, p. 243, 2017.

[19] S. Duval and R. Tweedie, "Trim and fill: a simple funnel-plotbased method of testing and adjusting for publication bias in meta-analysis," Biometrics, vol. 56, no. 2, pp. 455-463, 2000.

[20] L. Barrubés, N. Babio, N. Becerra-Tomás, N. Rosique-Esteban, and J. Salas-Salvadó, "Association between dairy product consumption and colorectal cancer risk in adults: a systematic review and meta-analysis of epidemiologic studies," Advances in Nutrition, vol. 10, pp. S190-s211, 2019.

[21] S. C. Larsson, L. Bergkvist, J. Rutegard, E. Giovannucci, and A. Wolk, "Calcium and dairy food intakes are inversely associated with colorectal cancer risk in the Cohort of Swedish Men," The American Journal of Clinical Nutrition, vol. 83, no. 3, pp. 667-673, 2006.

[22] E. Kampman, E. Giovannucci, P. Van't Veer et al., "Calcium, vitamin D, dairy foods, and the occurrence of colorectal adenomas among men and women in two prospective studies," American Journal of Epidemiology, vol. 139, no. 1, pp. 16-29, 1994.

[23] E. Kesse, M.-C. Boutron-Ruault, T. Norat, E. Riboli, and F. Clavel-Chapelon, "Dietary calcium, phosphorus, vitamin
$\mathrm{D}$, dairy products and the risk of colorectal adenoma and cancer among French women of the E3N-EPIC prospective study," International Journal of Cancer, vol. 117, no. 1, pp. 137-144, 2005.

[24] X. Zheng, K. Wu, M. Song et al., "Yogurt consumption and risk of conventional and serrated precursors of colorectal cancer," Gut, vol. 69, no. 5, pp. 970-972, 2020.

[25] M.-C. Boutron, J. Faivre, P. Marteau, C. Couillault, P. Senesse, and V. Quipourt, "Calcium, phosphorus, vitamin D, dairy products and colorectal carcinogenesis: a French case-control study," British Journal of Cancer, vol. 74, no. 1, pp. 145-151, 1996.

[26] B. Diergaarde, E. W. Tiemersma, H. Braam et al., "Dietary factors and truncatingAPC mutations in sporadic colorectal adenomas," International Journal of Cancer, vol. 113, no. 1, pp. 126-132, 2005.

[27] S. Jafari Nasab, M. Ghanavati, A. Bahrami et al., "Dietary nutrient patterns and the risk of colorectal cancer and colorectal adenomas: a case-control study," European Journal Cancer Prevention, vol. 30, 2020.

[28] V. Karagianni, E. Merikas, F Georgopoulos et al., "Risk factors for colorectal polyps: findings from a Greek case-control study," Revista medico-chirurgicala a Societatii de Medici si Naturalisti din Iasi, vol. 114, no. 3, pp. 662-670, 2010.

[29] G. A. Kune, S. Kune, A. Read, K. MacGowan, C. Penfold, and L. F. Watson, "Colorectal polyps, diet, alcohol, and family history of colorectal cancer: a case-control study," Nutrition and Cancer, vol. 16, no. 1, pp. 25-30, 1991.

[30] S. B. Rifkin, F. M. Giardiello, X. Zhu et al., "Yogurt consumption and colorectal polyps," The British Journal of $\mathrm{Nu}$ trition, vol. 124, pp. 1-12, 2020.

[31] P. Senesse, M.-C. Boutron-Ruault, J. Faivre, N. Chatelain, C. Belghiti, and S. Méance, "Foods as risk factors for colorectal adenomas: a case-control study in Burgundy (France)," Nutrition and Cancer, vol. 44, no. 1, pp. 7-15, 2002.

[32] C. Y. Um, V. Fedirko, W. D. Flanders, S. E. Judd, and R. M. Bostick, "Associations of calcium and milk product intakes with incident, sporadic colorectal adenomas," $\mathrm{Nu}$ trition and Cancer, vol. 69, no. 3, pp. 416-427, 2017.

[33] P. A. Wark, W. Van der Kuil, J. Ploemacher et al., "Diet, lifestyle and risk of K-ras mutation-positive and -negative colorectal adenomas," International Journal of Cancer, vol. 119, no. 2, pp. 398-405, 2006.

[34] N. Keum and E. Giovannucci, "Global burden of colorectal cancer: emerging trends, risk factors and prevention strategies," Nature Reviews Gastroenterology \& Hepatology, vol. 16, no. 12, pp. 713-732, 2019.

[35] V. Conteduca, D. Sansonno, S. Russi, and F. Dammacco, "Precancerous colorectal lesions," International Journal of Oncology, vol. 43, no. 4, pp. 973-984, 2013.

[36] W. B. Strum, "Colorectal adenomas," New England Journal of Medicine, vol. 374, no. 11, pp. 1065-1075, 2016.

[37] N. Keum, D. Aune, D. C. Greenwood, W. Ju, and E. L. Giovannucci, "Calcium intake and colorectal cancer risk: dose-response meta-analysis of prospective observational studies," International Journal of Cancer, vol. 135, no. 8, pp. 1940-1948, 2014.

[38] H. L. Newmark, M. J. Wargovich, and W. R. Bruce, "Colon cancer and dietary fat, phosphate, and calcium: a hypothesis," Journal of the National Cancer Institute, vol. 72, no. 6, pp. 1323-1325, 1984.

[39] S. A. Lamprecht and M. Lipkin, "Cellular mechanisms of calcium and vitamin $\mathrm{D}$ in the inhibition of colorectal 
carcinogenesis," Annals of the New York Academy of Sciences, vol. 952, no. 1, pp. 73-87, 2001.

[40] M. Pufulete, "Intake of dairy products and risk of colorectal neoplasia," Nutrition Research Reviews, vol. 21, no. 1, pp. $56-67,2008$.

[41] S. A. Lamprecht and M. Lipkin, "Chemoprevention of colon cancer by calcium, vitamin D and folate: molecular mechanisms," Nature Reviews Cancer, vol. 3, no. 8, pp. 601-614, 2003.

[42] T. U. Ahearn, A. Shaukat, W. D. Flanders, R. E. Rutherford, and R. M. Bostick, "A randomized clinical trial of the effects of supplemental calcium and vitamin D3 on the APC/ $\beta$-Catenin pathway in the normal mucosa of colorectal adenoma patients," Cancer Prevention Research, vol. 5, no. 10, pp. 1247-1256, 2012.

[43] P. S. Dulai, S. Singh, E. Marquez et al., "Chemoprevention of colorectal cancer in individuals with previous colorectal neoplasia: systematic review and network meta-analysis," BMJ, vol. 355, 2016.

[44] S. Rezac, C. R. Kok, M. Heermann et al., "Fermented foods as a dietary source of live organisms," Frontiers in Microbiology, vol. 9, p. 1785, 2018.

[45] J. R. Buendia, Y. Li, F. B. Hu et al., "Regular yogurt intake and risk of cardiovascular disease among hypertensive adults," American Journal of Hypertension, vol. 31, no. 5, pp. 557-565, 2018.

[46] M. L. Marco, D. Heeney, S. Binda et al., "Health benefits of fermented foods: microbiota and beyond," Current Opinion in Biotechnology, vol. 44, pp. 94-102, 2017.

[47] A. P. Bhatt, M. R. Redinbo, and S. J. Bultman, "The role of the microbiome in cancer development and therapy," CA: $A$ Cancer Journal for Clinicians, vol. 67, no. 4, pp. 326-344, 2017.

[48] Y. Zhang, X. Yu, E. Yu et al., "Changes in gut microbiota and plasma inflammatory factors across the stages of colorectal tumorigenesis: a case-control study," BMC Microbiol, vol. 18, no. 1, p. 92, 2018.

[49] W. Fong, Q. Li, and J. Yu, "Gut microbiota modulation: a novel strategy for prevention and treatment of colorectal cancer," Oncogene, vol. 39, no. 26, pp. 4925-4943, 2020.

[50] S. U. S. McGuire, "Department of agriculture and U.S. department of health and human services, dietary guidelines for Americans, 2010. 7th edition, Washington, DC: U.S. government printing office, january 2011," Advances in Nutrition, vol. 2, no. 3, pp. 293-294, 2011.

[51] V. Pala, S. Sieri, F. Berrino et al., "Yogurt consumption and risk of colorectal cancer in the Italian European prospective investigation into cancer and nutrition cohort," International Journal of Cancer, vol. 129, no. 11, pp. 2712-2719, 2011.

[52] P. Ambalam, M. Raman, R. K. Purama, and M. Doble, "Probiotics, prebiotics and colorectal cancer prevention," Best Practice \& Research Clinical Gastroenterology, vol. 30, no. 1, pp. 119-131, 2016.

[53] T. Akhavan, B. L. Luhovyy, S. Panahi, R. Kubant, P. H. Brown, and G. H. Anderson, "Mechanism of action of pre-meal consumption of whey protein on glycemic control in young adults," The Journal of Nutritional Biochemistry, vol. 25, no. 1, pp. 36-43, 2014.

[54] T. Akhavan, B. L. Luhovyy, P. H. Brown, C. E. Cho, and G. H. Anderson, "Effect of premeal consumption of whey protein and its hydrolysate on food intake and postmeal glycemia and insulin responses in young adults," The American Journal of Clinical Nutrition, vol. 91, no. 4, pp. 966-975, 2010. 